\title{
GCU
}

Glasgow Caledonian

University

University for the Common Good

\section{Acoustic analysis and mood classification of pain-relieving music}

Knox, Don; Beveridge, Scott; Mitchell, Laura A.; Macdonald, Raymond A.R.

Published in:

Journal of the Acoustical Society of America

DOI:

$10.1121 / 1.3621029$

Publication date:

2011

Document Version

Publisher's PDF, also known as Version of record

Link to publication in ResearchOnline

Citation for published version (Harvard):

Knox, D, Beveridge, S, Mitchell, LA \& Macdonald, RAR 2011, 'Acoustic analysis and mood classification of painrelieving music', Journal of the Acoustical Society of America, vol. 130, no. 3, pp. 1673-1682.

https://doi.org/10.1121/1.3621029

\section{General rights}

Copyright and moral rights for the publications made accessible in the public portal are retained by the authors and/or other copyright owners and it is a condition of accessing publications that users recognise and abide by the legal requirements associated with these rights.

Take down policy

If you believe that this document breaches copyright please view our takedown policy at https://edshare.gcu.ac.uk/id/eprint/5179 for details of how to contact us. 


\title{
Acoustic analysis and mood classification of pain-relieving music
}

\author{
Don Knox ${ }^{\mathrm{a})}$ and Scott Beveridge \\ School of Engineering and Computing, Glasgow Caledonian University, Cowcaddens Road, \\ Glasgow, G4 OBA, United Kingdom \\ Laura A. Mitchell \\ Department of Psychology, Bishop's University, 2600 College Street, Sherbrooke, Quebec J1M 1Z7, Canada
}

\author{
Raymond A. R. MacDonald \\ Department of Psychology, Glasgow Caledonian University, Cowcaddens Road, Glasgow, \\ G4 OBA, United Kingdom
}

(Received 24 March 2011; revised 1 July 2011; accepted 5 July 2011)

\begin{abstract}
Listening to preferred music (that which is chosen by the participant) has been shown to be effective in mitigating the effects of pain when compared to silence and a variety of distraction techniques. The wide range of genre, tempo, and structure in music chosen by participants in studies utilizing experimentally induced pain has led to the assertion that structure does not play a significant role, rather listening to preferred music renders the music "functionally equivalent" as regards its effect upon pain perception. This study addresses this assumption and performs detailed analysis of a selection of music chosen from three pain studies. Music analysis showed significant correlation between timbral and tonal aspects of music and measurements of pain tolerance and perceived pain intensity. Mood classification was performed using a hierarchical Gaussian Mixture Model, which indicated the majority of the chosen music expressed contentment. The results suggest that in addition to personal preference, associations with music and the listening context, emotion expressed by music, as defined by its acoustical content, is important to enhancing emotional engagement with music and therefore enhances the level of pain reduction and tolerance. (C) 2011 Acoustical Society of America. [DOI: 10.1121/1.3621029]
\end{abstract}

PACS number(s): 43.75.Cd, 43.75.Xz, 43.75.Zz [DD]

Pages: $1673-1682$

\section{INTRODUCTION}

Responses evoked by listening to music in a variety of contexts have been the focus of a growing body of research. Examples include perception of waiting time (North et al., 1999), behavior during everyday activities such as shopping (North and Hargreaves, 2008), driving (Dibben and Williamson, 2007), and playing video games (Cassidy and MacDonald, 2009). The acoustical content of the music is rarely examined in these studies, rather description of the music signal is typically constrained to measures such as tempo (Cassidy and MacDonald, 2010; North and Hargreaves, 2008), complexity (Lamont and Webb, 2010), or arousal potential (North and Hargreaves, 1999). Recent years have seen a trend toward studies concentrating upon the effects of listening to preferred music chosen by the participant in variety of everyday activities (see Miell et al., 2005; North and Hargreaves, 2008). Music plays a significant role in everyday activity, is primarily goal-directed (Sloboda and O'Neill 2001; North et al., 2004), and has been found to influence behavior in a variety of contexts (Miell et al., 2005). In studies using preferred music, emphasis is placed upon the participant's relationship with the music rather than the content of the music itself. These studies have illustrated that preferred music choice is diverse, in that it varies widely in

\footnotetext{
a) Author to whom correspondence should be addressed. Electronic mail: d.knox@gcu.ac.uk
}

terms of artist, tempo, and genre. However, the acoustical properties of the music in question are unknown and have not been examined in detail. Thus the possible influence of acoustical parameters upon the observed beneficial effects of music listening has not been examined.

An important and growing area of this research is concerned with the effect of music listening on pain relief. Studies on the analgesic effects of music have been carried out in both clinical and laboratory settings. A systematic review of these studies concludes that music listening, in the appropriate context, can reduce pain intensity levels and reduce the opioid requirements of participants with postoperative pain (Cepeda et al., 2006). The effects of music on perception of pain intensity in the studies included in the review are small, and the authors conclude the specific use of music for pain relief in clinical settings is not clear. However, the positive effects of music listening upon a variety of physiological and psychological effects of pain in a clinical context have been demonstrated. For example reduction in blood pressure (Updike, 1990), reduction of nausea and vomiting (Madson and Silverman, 2010), reduction of pain-related distress (Huang et al., 2010). Music listening may mitigate the negative affective experiences associated with pain in addition to reducing the sensation of pain (Mitchell and MacDonald, 2006).

Much of the research into causal mechanisms in pain relieving effects of music has developed from gate control and pain neuromatrix theory (Melzack and Wall, 1965; 
Melzack, 1993, 1999). This theory suggests the flow of nerve impulses from the peripheral nerve fibers to the central nervous system can be modulated by cognitive and emotional processes, and this has generated a vast body of literature on potential interventions to modulate attention, emotion or perception of control over pain. Two main theoretical explanations for music's effect on pain have been proposed in previous studies (Mitchell et al., 2006). The first is the ability to use music freely at any time while in pain-providing a feeling of control and the ability to do something to counteract the negative experience (Brown et al., 1989). The second is distraction of attention from the pain (Fauerbach et al., 2002), which suggests distracting outside tasks leave limited mental resources for pain perception (Shiffrin, 1988). Later research has suggested that emotional engagement with a stimulus creates the strongest distraction (Robinson, 1998). This emotional connection may be moderated by factors surrounding the music itself. Evidence of pain reduction while listening to pleasantly valenced music has highlighted that positive emotional induction may play an underpinning role in music's effect (Roy et al., 2008). Emotional factors beyond the music itself are explored by an increasing number of music psychology studies. These highlight the emotional component of distraction, describing the "associative" context of listening as personal memories and meanings connected with the music (Miell et al., 2005). These issues are explored by an increasing number of studies into participants' preferred music, as opposed to music chosen for its supposed relaxing or calming effects. Music chosen by participants in studies examining pain tolerance resulted in reduced anxiety, increased relaxation (Davis and Thaut, 1989), and positive effects on pain tolerance and perception (Helmet and Hertel, 1993; Jacobson, 1999; Perlini and Viita, 1996). Beneficial effects have been linked to increased levels of involvement with music, which in turn depends upon familiarity and past associations with the music, and cultural context (MacDonald et al., 2003). The efficacy of preferred music in this context has been supported by a number of lab-based studies using cold pressor techniques, where the preferred music listening condition resulted in the following.

(1) Increased pain tolerance and perceived control over pain when compared to white noise and specifically chosen "relaxing" music (Mitchell and MacDonald, 2006).

(2) Significant increases in pain tolerance in comparison to an arithmetic distraction task, and significantly increased perceived control in comparison to humor as an engaging distraction (Mitchell et al., 2006).

(3) Significantly increased tolerance and perceived control over pain, decreased anxiety compared with both visual distraction and silence, and reduced pain intensity when compared with silence (Mitchell et al., 2008a).

(4) Significantly greater tolerance of pain compared to silence and focusing on pain, and significantly greater perceived control than when focusing on pain (Mitchell, 2009).

These findings have been replicated in other contexts, such as during kidney dialysis (Pothoulaki et al., 2008).
The content of the music used in these studies is rarely emphasized. Researchers studying preferred music and pain relief suggest that structural features alone cannot explain emotional reactions in the listener (Mitchell et al., 2006). Rather external factors play a key role in the emotional meaning of music (MacDonald and Miell, 2000), and that personal meaning and memories associated with the music affect our emotional connection with it (Miell et al., 2005). The increased therapeutic efficacy of preferred music when compared to experimenter-selected anxiolytic music suggests that beneficial effects are dependent upon personal associations and emotional engagement with the music, and that this effect is independent of musical features present in supposed calming or relaxing music. Informal post hoc examination of the music chosen by participants in these experiments seems to support this assertion, in that it varies widely in terms of artist, tempo, and genre. It has been suggested that listening to preferred music may make compositions with widely varying structural features "functionally equivalent" (Mitchell et al., 2008a). Specifically, it has been suggested that participants select music with different structural features to achieve the same psychological ends, namely, the reduction of pain and anxiety. The literature on preferred music and pain relief supports the importance of personal preference, associations with music and the listening context. However, the music used in these studies has not been subject to detailed analysis, and there is therefore no evidence to support the assertion that beneficial effects are independent of the structure of the music. The focus of the current paper is to examine the possible influence of structure in music chosen for pain relief.

Given that the effectiveness of musical stimuli to distract from pain is related to emotional engagement with the stimuli, it follows that the content of the music is important in this regard. Music content has long been the focus of research into music and emotion, with particular emphasis on the effect of particular structural elements upon the emotions of the listener. Listeners display a common emotional response to a wide range of stimuli (Ekman and Friesen, 1998), and there is marked agreement in the emotion being expressed by music between different listeners (Krumhansl, 2002). This suggests cultural differences may not be so strong as to make emotional categorization culturespecific. The most common goal for music listening is to influence emotions (Sloboda and O'Neill, 2001; Juslin and Laukka, 2004). Some research has focused upon strong emotional responses to music, also known as "thrills" or "chills" (Sloboda, 1991; Panksepp, 1995; Gabrielsson, 2001). Emphasis has also been placed upon specific music performance parameters important to causing emotional reactions (Scherer and Zentner, 2001; Gabrielsson and Lindström, 2001; Juslin, 2000; Schoonderwaldt et al., 2002; Chapin et al., 2008). Others have examined the concept of musical expectation (Meyer, 1956), the assumption that while listening to music an individual will form expectancies about its continuation. Corresponding emotional reactions are evoked in the listener if presuppositions are violated (Narmour, 1990; Huron, 2006). Despite the recent popularity of research focused on this subject there remains significant 
debate about the nature of musical emotions. There is a clear distinction between emotion experienced by the listener and perception of emotion expressed by the music (Gabrielsson, 2002), but there is disagreement on whether or not music actually evokes emotions in the listener (Konecni, 2008). This conflict has led to the synthesis of a holistic, multidimensional framework for studying the induction of emotion by listening to music-the BRECVEM model-which aims to examine the underlying psychological mechanisms which affect listeners' emotional response to music (Juslin and Västfjäll, 2008). Three of the key mechanisms described in the model are dependent to varying degrees upon the structure of the music. These are brain stem reflex, where basic acoustical characteristics of music cause corresponding activation of the central nervous system; emotional contagion, which suggest the listener mimics the mood expressed by a piece of music; and musical expectancy. The precise mechanisms for induction of emotion by music are not yet fully understood, and these mechanisms have been proposed as a basis for future empirical research into induction of emotion through music listening. However, the content and structure of music plays a significant role in the communication of emotion by music.

The two-dimensional energy-stress model of Thayer (1989) is a common method of both measuring the emotion expressed by music, and explaining participants' emotional responses to music (see, for example, Ritossa and Rickard, 2004). This model is based upon Russell's circumplex model of affect (Russell, 1980) and is adapted for music emotion. In Thayer's model, the axes are energy (arousal) and stress (valence), with four quadrants equating to music which is content, depressed, exuberant, and anxious/frantic (see Fig. 1). The horizontal axis represents valence (negative to positive from left to right), and the vertical axis represents intensity (low to high).

The labeling of the axes varies, and the adjectives describing the four quadrants, or clusters, can differ but retain a similar meaning. It is also possible to overlay spe-

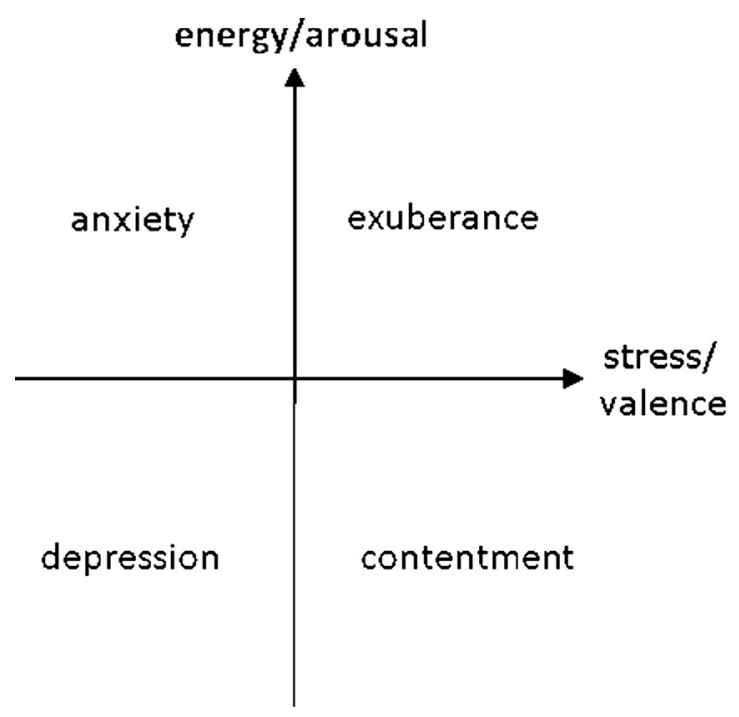

FIG. 1. Circumplex stress/energy model showing the four mood clusters; contentment, depression, exuberance and anxiety. cific mood adjectives relating to varying points in the four quadrants (Picard, 1997; Juslin, 2000; Hevner, 1935). The circumplex model has been used extensively in recent research in the field of Music Information Retrieval (MIR) which has focused upon automatic classification of the emotion expressed by music (Li and Ogihara, 2003; Lu et al., 2006; Yang et al., 2008; Knox et al., 2008; Eerola and Vuoskoski, 2011). These methods are based upon detailed analysis of digital music files and extraction of a range of acoustical parameters which correlate with performance features important to the expression of emotion in music (Juslin, 2001). Lu et al. (2006) use measures of signal intensity, timbre, and rhythm as a basis for mood classification. These include spectral intensity, spectral shape, rhythm, tempo, and rhythm regularity. Yang et al. (2008) extract further features, including those utilized by Tzanetakis and Cook (2002), and those produced by the PsySound program (Cabrera, 1999), which include measures of loudness, dissonance, and pitch. The circumplex model is often preferred to individual adjectives in automatic mood classification due to the relative ease with which signal analysis parameters may be mapped to the two axes. For example, signal intensity features can determine a song's position in the energy/arousal axis, and timbre and rhythm features determine placement on the stress/valence axis (Lu et al., 2006). Two-dimensional classification models have been criticized for their ambiguity in classifying music in terms of only four mood quadrants, and that emotion states may vary widely within each quadrant. This has led to the implementation of three-dimensional valence, energy-arousal, and tension-arousal models, which aim to more accurately predict individual emotions (Eerola et al., 2009).

The technological developments in MIR allow for analysis of the content of digital music files in unprecedented detail, producing a wealth of acoustical signal parameters. These features may also form the basis for subsequent statistical analysis and classification in terms of the emotion music expresses. A key research question posed in this paper is whether there is a relationship between the structural content of preferred music chosen for pain relief and its positive effect in these experiments. There is no a priori hypotheses about the likely effects of specific acoustic properties of the music, or the age and gender of the participants. Rather the aim of the research presented here is to examine whether the structure of music, and the emotion expressed by it, are factors influencing the beneficial effects of music chosen for pain relieving purposes.

\section{METHODS}

Music chosen by participants in three experiments which studied the effects of preferred music on pain was subject to post hoc analysis (Mitchell et al., 2008a; Mitchell et al., 2008b; Mitchell, 2009). In each experiment a commercially available cold pressor was used (Fig. 2), which requires the participant to place their hand in cold, circulating water. This technique allows for a precise and controllable temperature to be applied and ensures replicable results (Mitchell et al., 2004). A repeated measures design was 


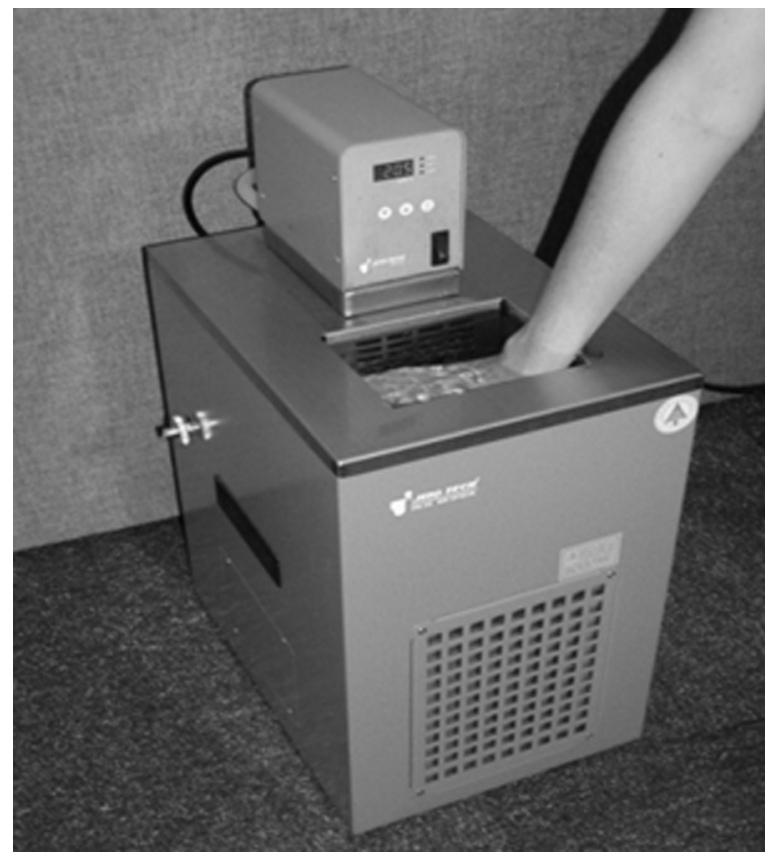

FIG. 2. Cold Pressor used in the pain experiments.

used, with each participant undergoing three cold pressor trials. Stimuli were presented in counterbalanced order, and test conditions in the experiments were as follows.

(1) Preferred music, silence, and visual distraction (Mitchell et al., 2008a). Visual distraction took the form of a painting chosen by the participant from a choice of 15 popular artworks. This study involved 80 participants.

(2) Preferred music listening and mental arithmetic distractions compared to silence. This study involved 72 participants (Mitchell et al., 2008b).

(3) Silence, focusing on pain (where participants paid attention to the painful sensation and how it changed over time), and listening to chosen music. 55 participants (Mitchell, 2009).

In each case stimuli were delivered via headphones and set by participants at a "comfortable" level. Participants knew the nature of the experiment before participating, and were asked simply to bring some music. Reasons given by participants for their choice of music in a similar, earlier study indicate that "favorite" music was chosen in $87 \%$ of cases (Mitchell and MacDonald, 2006). Dependent variables were the same in each experiment: tolerance time (the amount of time, in seconds, the participant kept their hand in the water); pain intensity (as measured via visual analog scale and pain rating index (Melzack, 1975); perceived control rating on a visual analog rating scale (Perlini and Viita, 1996); and anxiety measured via the State-Trait Anxiety Inventory (Spielberger, 1983).

There are two key aims in analysis of the music from these experiments. First to examine the structure of the preferred music in comparison to pain measurements taken in the experiments, with the aim of establishing whether any structural feature may have influenced its efficacy in mitigating the effects of pain. This analysis includes content-based mood classification of the preferred music in order to examine whether there is any commonality in the emotion expressed by music chosen by the participants. Second, a comparison is made between the preferred music from the experiments and a corpus of a broad range of music, with the aim of establishing whether there are any overarching structural differences between music shown to have beneficial effects in the experiments. This corpus consists of 160 clips of music representing a selection of genres matching the music chosen by the participants in the pain experiments (classical, alternative, pop, jazz, and rock).

Data was gathered for a selection of participants from the above experiments, including pain measurement data and the music which the participants chose. The selection of data for analysis is based on the measurements taken during the pain experiments. In addition to the gender and age of participants, these measurements are the following.

(1) Tolerance time (TOLdiff). Tolerance time is the time from immersion of the hand to the point when participants can no longer tolerate the stimulus and withdraw the hand. Measured in seconds by stopwatch. The TOLdiff parameter is produced by calculating the difference in tolerance time between the silence and preferred music conditions.

(2) Pain intensity rating on a $100-\mathrm{mm}$ visual analog scale (VASdiff), on which participants are asked to make a mark between no discomfort and worst possible discomfort. The VASdiff parameter is produced by calculating the difference in rated discomfort levels between the silence and preferred music conditions.

(3) A 100-mm perceived control visual analog rating scale (PCdiff), on which participants mark the extent to which they felt they could successfully manage or alter the pain experience between "not at all" and "completely." The $P C$ diff parameter is produced by calculating the difference in perceived control between the silence and preferred music conditions.

(4) A short form of the Spielberger State Anxiety Questionnaire with a rating given between 1 (strongly agree) and 7 (strongly disagree) for items "I felt calm," "I felt relaxed," "I felt tense," "I felt comfortable," and "I felt anxious." The ANXdiff measure is produced by calculating the difference in rated anxiety levels between the silence and preferred music conditions.

These studies have demonstrated the efficacy of preferred music in mitigating the effects of pain, and particularly its effect on increasing pain tolerance. The music which was shown to be most effective in the experiments is chosen for analysis, and selection is based on two criteria. First, the tolerance increase from the control condition to preferred music listening condition has to be positive for the music to be selected. Pain tolerance time is a key objective measure from the pain experiments, the other measures being self-rated, and it is felt this measure must be increased by listening to the preferred music for it to be seen as effective. The second selection criteria is the overall score for all four pain measurements. This is calculated by combining the four measurements into one measure for "total therapeutic 
value" (TTV). Selected tracks had to have a TTV score in the top half of the normally distributed scores for all tracks from the pain experiments, and also a positive tolerance difference. Therefore, the criteria for selection were the greatest increase in tolerance time compared to control, and the highest TTV. In total, data for 72 participants is selected, and 130 participants are excluded. The means and standard deviations for all four pain measures and TTV for the selected and non-selected participants under the preferred music listening condition are shown in Table I. This selection process distinguishes between music that was simply preferred by the participants, and music which is preferred but has also been shown to have the most therapeutic effect.

Preferred music choices are truncated to $30 \mathrm{~s}$ clips for subsequent analysis. Although it has been established that listeners can be affected by listening to very short music clips (Bigand et al., 2005), this duration is in line with previous music analysis implementations which use short clips of between 10 and $30 \mathrm{~s}$ length in order to reduce the analysis and calculation burden, or to ensure homogeneity of mood across the duration of the clip (Li and Ogihara, 2003; Jiang et al., 2002; Lu et al., 2006). In this instance, the clip duration is chosen to reflect the music which participants actually heard while their hand was in the water. The audio feature extraction stage is carried out using two separate analysis feature sets. First a set of intensity, timbre, and rhythm features shown to be effective in the automatic mood classification of music ( $\mathrm{Lu}$ et al., 2006), and important to emotion expressed by music (Gabrielsson and Lindström, 2001). Second, use is made of the MIR toolbox of Lartillot et al. (2008b). The extended set of features produced by this toolbox relate to musical dimensions such dynamics, rhythm, timbre, pitch, tonality, mode, and high level statistical and structure parameters noted as being of potential importance to improved music mood detection (Lu et al., 2006). Several measurements of signal timbre are calculated from frequency spectra. These include spectral centroid (a measure of the shape of the spectrum) and roll-off (the frequency below which $85 \%$ of the spectral energy is concentrated and thus a measure of brightness), Mel-Frequency Cepstral Coefficients (MFCC, a compact representation of the spectral envelope based on an approximation of human hearing frequency response) and spectral flux (fluctuation in the spectrum over time). For a detailed description of these parameters see Tzanetakis and Cook (2002). Sensory dissonance (perceived roughness) is assessed by the degree of spectral beating effects (Terhardt, 1974), and tonality is assessed by examining pitch class profiles (Gomez and Herrera, 2004). A range of rhythm information is provided

TABLE I. Pain measurement means and standard deviations for selected and non-selected music.

\begin{tabular}{lcccccc}
\hline \hline & $\mathrm{N}$ & TOLdiff & VASdiff & ANXdiff & PCdiff & TTV \\
\hline Selected & 72 & 87.4 & 20.9 & 3.4 & 23.7 & 135.7 \\
& & $\mathrm{SD}=81.4$ & $\mathrm{SD}=22.3$ & $\mathrm{SD}=5.1$ & $\mathrm{SD}=22.5$ & $\mathrm{SD}=106.7$ \\
Not selected & 130 & 9.4 & 4.4 & 1.5 & 4.8 & 20.1 \\
& & $\mathrm{SD}=53.1$ & $\mathrm{SD}=18$ & $\mathrm{SD}=5.4$ & $\mathrm{SD}=21.8$ & $\mathrm{SD}=79.4$ \\
\hline \hline
\end{tabular}

through decomposition of the spectrogram through a bank of auditory filters, and subsequent spectrum estimation of each band (Pampalk et al., 2002). In addition to being associated with music performance parameters, these primarily acoustical features are linked to low-level, primitive neural processing (Levitin, 2006).

The first stage of analysis involves comparison of audio signal analysis features with pain measurement parameters. For this first comparison, a subset of the rhythm, timbre, and dynamics features is chosen based upon their theoretical relevance to the perception of music, and their successful use in previous studies of music and emotion. These features form the basis for statistical analysis to establish whether there are any significant relationships between the parameter set and the pain measures from the experiments. The initial parameter set consists of the following.

(1) Tempo, rhythm strength, rhythm regularity, and average onset frequency, based on the observation that these parameters are important to expression of musical emotion (Gabrielsson and Lindström, 2001; Lu et al., 2006; Juslin, 2000). Rhythm regularity refers to the strength of peaks in the autocorrelation function, more precisely the average strength of the peaks and the ratio between the average peak strength and trough strength. If the rhythm is regular and unchanging this ratio is higher. Average onset frequency relates to the perceived rate of the rhythmic element of the music. This is distinct from tempo, which simply measures the main beat pulse. Average onset frequency is calculated as the ratio between the number of onsets and the corresponding time duration (Lu et al., 2006). The larger this value it is, the faster the performance feels for a given tempo.

(2) Measures of timbre include rolloff, brightness, and roughness. Timbral features such as these are correlated with articulation, and thus important to emotion expression (Lu et al., 2006). Brightness is a measure of high frequency energy, and measures the percentage of energy above a given point in frequency, in this case 1.5 $\mathrm{kHz}$ (Juslin, 2000). Roughness is a measure of sensory dissonance computed by examining peaks in the spectrum, and taking the average of the dissonance between all possible pairs of peaks (see Sethares, 1998).

(3) Dynamics features RMS energy and low energy. These features have been shown to be consistent with acoustic cues in emotional expression, and important in distinguishing between mood clusters in the circumplex model (Juslin, 2000; Lu et al., 2006). RMS energy is the average intensity over the duration of the music clip, taken as the root average of the square of the amplitude. Low energy is a measure of how consistent the energy is across the clip duration. If this value is high large parts of the music are of low-intensity, and higher intensity parts are more widely distributed. If it is lower, the signal intensity is more consistent around the average intensity (Lartillot et al., 2008a).

The second analysis stage utilizes a verbose set of analysis parameters produced by the MIRfeatures function of the MIR Toolbox (Lartillot et al., 2008b). A statistical mapping 
is performed between the extracted features and the pain measurements from the experiments using stepwise linear regression. Lilliefors test is used to detect non-normality in the feature set (at a significance level of 5\%), and any nonnormal data transformed by the Box-Cox power transformation, thus ensuring feature set distributions are sufficiently normal (Box and Cox, 1964). Features still not considered to be normalized after this process (at a significance level of $1 \%$ ) are discarded from further analysis. These processes are encapsulated in the MIRmap function of the MIR toolbox (Lartillot et al., 2008a). The results of this mapping indicates which signal analysis features are correlated with pain measurements taken during the experiments.

The third stage of analysis is content-based mood classification of the music chosen by the participants. This stage of analysis utilizes a hierarchical categorical mood classification algorithm shown to be successful in automatic mood classification (Lu et al., 2006). The mood classification process is driven by analysis of structural elements of the music. Thus the process takes into account the structural elements in music chosen for pain relief, and their contribution to the mood expressed by the music. The process utilizes a Gaussian Mixture Model (GMM), where the content of the preferred music is compared statistically to a "ground truth" of music annotated for mood by an expert panel in subjective listening tests using the circumplex model shown in Fig. 1. This is a corpus of 160 music clips gathered by the authors, and selected from a range of genres and recording eras matching those chosen by the participants in the pain experiments. The panel consists of four musicians with extended experience of music analysis. In line with similar studies of mood classification, clips were only selected for the corpus if all members of the panel agreed on the mood of the clip, and there was no ambiguity in the perceived mood (Lu et al., 2006). The basis for classification is a series of timbre, rhythm, and intensity parameters.

In the original implementation (Lu et al., 2006) intensity features were noted to be important to distinguishing between mood clusters on the vertical axis of Thayer's circumplex model (i.e., between exuberant/anxious-frantic and content/depressed). Timbre features are considered to be important to discriminating different moods. For instance greater spectral energy is present in high frequency spectral subbands for exuberant music than for depressed music. Spectral flux is taken as an indicator of articulation-noted to be an important factor in emotional expression (Juslin, 2000). Octave-based spectral contrast is a measure of the distribution of peaks and troughs in the spectrum and reflects the harmonic/inharmonic distribution of spectral components. Rhythm strength, regularity, and tempo are chosen for their influence in expression of mood. For example music in the exuberant mood cluster tends toward fast, strong, and regular rhythms, whereas depressed music is slower and may have less distinct rhythm patterns (Liu et al., 2003). These features are then standardized (z-scores), and then the Karhunen-Loeve transform is applied for each feature set independently in order to remove correlation among the features (see Watanabe, 1965). These processes result in the means and standard deviations of the extracted features being used as a basis for subsequent classification stages. The hierarchical classification model uses intensity features to determine the analyzed music's position on the energy/arousal axis. Timbre and rhythm features are used to determine position on the stress/valence axis. See Fig. 3 where group 1 refers to low activity/intensity (content/depressed) and group 2 refers to high activity/intensity (exuberant/anxious-frantic).

The GMM uses intensity, timbre, and rhythm parameters extracted from the 160 music clip corpus music as a basis for mood classification, with 40 clips representing each circumplex mood cluster. The first stage of classification is based on intensity features. This stage decides whether the analyzed music falls in the upper half of the circumplex model (anxious/frantic and exuberant) or lower half (content or depressed). The second stage uses timbre and rhythm features, to decide if low intensity music falls into the content or depressed clusters, and high intensity music into exuberant or anxious frantic clusters. Noting the varying importance of the role played by different performance cues in expressing different emotions (Juslin, 2000), the relative influence of the rhythm and timbre features is altered during this classification stage. The $\lambda$ operators in Fig. 3 are weighting factors set to reflect the relative importance of rhythm and timbre features in distinguishing between different mood clusters. For example, music which has a slower tempo with less prominent rhythm might be classified as falling into either of the content or depressed clusters. Content music tends toward higher brightness and is more harmonic than depressed music. Therefore, timbre features are important in distinguishing these two mood clusters.

\section{RESULTS}

There is significant negative correlation between participant age and ANXdiff, indicating older participants experienced less anxiety reduction. Age is significantly negatively correlated with RMS intensity, rolloff, and average onset

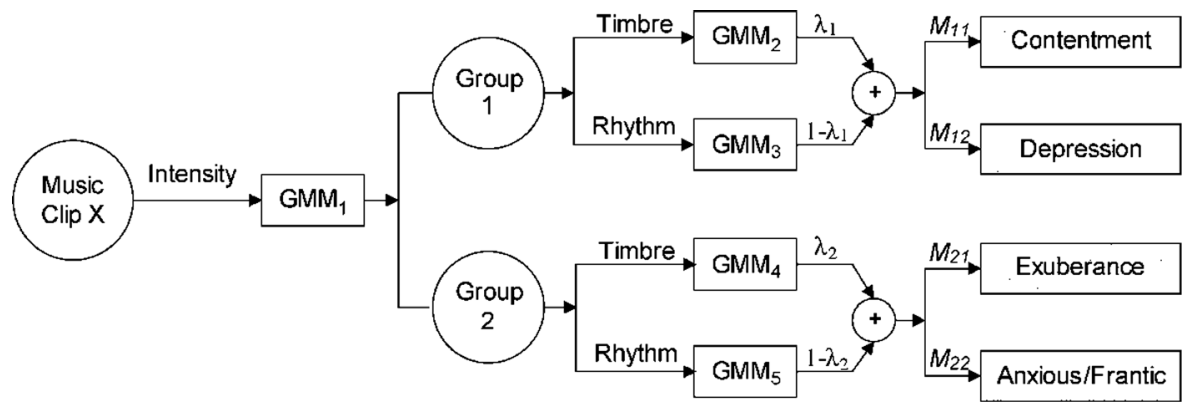

FIG. 3. Hierarchical Gaussian Mixture Model (from Lu et al., 2006). 
TABLE II. Factors correlating significantly with participant age.

\begin{tabular}{ccrrrc}
\hline \hline & & RMS & & $\begin{array}{c}\text { Average onset } \\
\text { frequency }\end{array}$ \\
\hline Age & Pearson Correlation & -0.254 & -0.251 & -0.254 & -0.302 \\
& Sig. (2-tailed) & 0.031 & 0.034 & 0.031 & 0.01 \\
\hline \hline
\end{tabular}

frequency (see Table II). These results indicate older participants chose music which is of lower intensity, contains less high frequency energy, and with less rapid or complex rhythm patterns. The mean age for those selected for this study is 28.2 [standard deviation $(\mathrm{SD})=11.2$ ], and 27.2 $(\mathrm{SD}=10.2]$ for those not selected.

Results for regressions using the verbose MIRfeatures parameter set indicate timbre features (spectral and tonal) are significantly correlated with pain measures from the experiments. This suggests that, within this data set, timbral features of the preferred music are most important to perception of pain intensity and the degree of pain tolerance. One parameter is strongly correlated negatively with pain tolerance time. This is chromagram peak position standard deviation $(r=-0.3306, p<0.01)$, which represents the variation in position of the most prominent peak in an unwrapped chromagram across the duration of the clip. The chromagram represents the distribution of the spectral energy with respect to the 12 possible pitch classes (see Gomez and Herrera, 2004). This result indicates that pain tolerance is greater for music which has less tonal (pitch) variation across the duration of the clip being analyzed. Features representing the distribution of energy in the spectrum and the rate of fluctuations in the spectrum over time are significantly correlated with pain tolerance and intensity. These are MFCC and $\delta$ MFCC parameters (see Lartillot et al., 2008b), which are representative of changes in music timbre and articulation. The mean spectral energy in the 12th MFCC band is significantly positively correlated with pain tolerance $(r=0.301$, $p=0.01)$. There is also significant positive and negative correlation between MFCC bands and perception of pain intensity. The standard deviation of energy levels in the $1 \mathrm{st}$ MFCC band are significantly negatively correlated with pain intensity $(r=-0.2765, p<0.05)$, as is mean energy in the 7 th MFCC band $(r=-0.2866, p<0.05)$. The feature most significantly correlated with pain intensity is Harmonic Change Detection Function (HCDF) periodicity entropy $(r=0.3126, p<0.01)$. HCDF is the flux of the tonal centroid, and is therefore a measure of the change in harmonic content over time (see Harte and Sandler, 2006). This value is small when there are less prominent changes of harmonic content in the analyzed music; therefore, this result suggests pain intensity levels are lower for music in which there may be less prominent chord changes, bass lines, or strong melodies.

The mood classification results show that preferred music chosen by participants falls predominantly in the "content" mood cluster (37.5\% of the clips analyzed). This indicates a tendency toward low arousal, and positive valence (see Table III). Chi square shows significant difference between mood clusters [chi(3) $=8.11, p<0.05]$.
TABLE III. Mood classification of music chosen during the pain experiments.

\begin{tabular}{lcc}
\hline \hline & Number of clips & \% of clips \\
\hline Mood cluster & 27 & \\
Content & 17 & 37.5 \\
Depressed & 18 & 23.6 \\
Exuberant & 10 & 25 \\
Anxious/Frantic & & 13.9 \\
Arousal/Valence & 44 & \\
Low arousal & 28 & 61.1 \\
High arousal & 44 & 38.9 \\
Positive valence & 28 & 62.5 \\
Negative valence & & 37.5 \\
\hline \hline
\end{tabular}

These results indicate that the music chosen by participants is of lower intensity and slower tempo, meaning classification tends toward the content/depressed end of the arousal/activity axis. The chosen music is also brighter, placing it toward the content/exuberant area of the circumplex model. Statistical comparison of the preferred music with the 160 clip corpus using one-way analysis of variance shows the preferred music is significantly brighter than the 160 clip corpus, as indicated by measures of spectral brightness $[F(231)=3.955, p<0.05]$ and mean spectral entropy $[F(231)=4.205, p<0.05]$. The mean value for modality, as measured by the MIR toolbox MIRmode function is significantly higher for preferred music $[F(231)=5.703$, $p=0.018]$. Higher values for this parameter indicate that the analyzed clip tends toward major mode. Thus, the preferred music is significantly more major than the 160 clip corpus.

\section{DISCUSSION}

Informal examination of music chosen by participants in studies of preferred music and pain have suggested thus far that there are no structural similarities, and that listening to preferred music may make compositions with widely varying structural features "functionally equivalent" (Mitchell et al., 2008a). The results presented in this paper represent some evidence that structural features of music chosen by participants play a role. Thus, structure should perhaps be considered alongside personal preference, associations with music, and the listening context in studies of music preference, in general, and specifically music chosen for pain relief.

Results show older participants chose music which is of lower intensity, contains less high frequency energy, and with less rapid or complex rhythm patterns. These results are consistent with previous research into age and music preference. For example, older participants have been shown to prefer decreased intensity levels (Smith, 1989), and younger listeners prefer faster tempi (LeBlanc et al., 1988; Drake et al., 2000).

Regression results show significant correlation between timbral and tonal analysis parameters and measures of pain tolerance and perceived intensity. No intensity or rhythm features produced by the MIRfeatures algorithm were significantly correlated. These results indicate spectra and timbral 
aspects of the chosen music were most important in affecting these pain measures - specifically tonal variation, prominence of melody or chord changes, and variations in timbre and articulation.

Statistical analysis using analysis of variance found no significant main effect for signal analysis parameters and measures from the pain studies. The data chosen for analysis was selected to represent the most effective selection of music as regards mitigating the effects of pain, based upon the measures taken during the experiments. One might not therefore expect stark contrasts between analysis parameters within this data set as regards their effect upon the pain measures. Therefore, future analysis of preferred music in this context might include preferred music choices that were minimally effective as regards mitigating the effects of pain.

From previous discussion, distraction from pain is a key mechanism underpinning the efficacy of music listening, and emotional engagement with the stimulus is important in this regard (MacDonald et al., 2003). Although the precise mechanisms for induction of emotion by music are not yet fully understood, the structure of music and the emotion it expresses cannot be ignored, as it is central to several mechanisms considered important to the future study of induction of emotion through music listening (Juslin and Västfjäll, 2008). Low level acoustical parameters and the emotion expressed by music relate to brain stem response and emotion induction mechanisms, respectively. The mood classification results show a significant difference in the emotion expressed by the preferred music chosen by the participants in the pain experiments with the majority expressing contentment. The mood classifications are based on analysis of music structure, and trends in the classification results are therefore indicative of general patterns in the acoustical content of the music. These trends are underlined in the analysis of variance results, which indicate the chosen music is brighter and more major in modality. Timbral measures, representative of articulation and the expression of musical emotion, are important to the placement of music on the valence axis of the circumplex model during mood classification. Regression results also show timbral and tonal parameters to have a significant effect upon measures of pain tolerance and perceived intensity. This suggests there is some effect for both low-level acoustical parameters and higher level music structure.

These results represent some evidence toward expressed emotion playing a role in the participants' choice of music for the pain experiments. This supports previous research into positive emotional induction and the effect of music listening (Roy et al., 2008), suggesting that the emotion expressed by a piece of music may be a factor affecting pain in two potential ways: the participant's emotional engagement with music evoking positive pleasant emotions that modify how pain is experienced, and through enhanced efficacy in distracting attention from pain.

There are several limitations to the study presented in this paper, due in the main to the limited availability of data for analysis. The results are based upon analysis of a relatively small sample size. The criteria for selection (positive tolerance time when compared to the control condition, and high overall therapeutic effect) resulted in analysis of 72 tracks from the identified pain experiments. The corpus of 160 tracks is also relatively small, although vetted by an expert panel and sufficient for the mood classification carried out in this study. Further comparison of music analysis data and pain parameters may be expanded by inclusion of data for minimally effective music choices. The music analysis excludes lyrical content, a factor which can improve emotion classification ratings (Chi et al., 2009). Authors of previous studies have noted that experimentally induced pain cannot fully replicate complex emotional responses resulting from clinical pain (Mitchell et al., 2006), and that studies into preferred music might ideally be based in this context.

\section{v. CONCLUSIONS}

This study examines music chosen by participants in previous laboratory experiments involving experimentally induced pain. The literature on preferred music and pain relief supports the importance of personal preference, associations with music, and the listening context. Results suggest that music content and structure may also play a role in the music choices made by participants and the efficacy of music listening for pain relief. Music chosen by participants predominantly expresses contentment, is generally brighter than other music, and more major in modality. Acoustical features are key to determining the emotion expressed by music, and timbral and tonal features are shown to be significantly correlated with measurements of pain tolerance and perceived pain intensity. It is suggested that the acoustical content and emotion expressed by a piece of music contribute toward the participant's emotional engagement with music and enhances distraction from pain.

Bigand, E., Filipic, S., and Lalitte, P. (2005). "The time course of emotional responses to music," Ann. N. Y. Acad. Sci. 1060, 429-437.

Box, G. E. P., and Cox, D. R. (1964). "An analysis of transformations," J. R. Stat. Soc. Ser. B 26, 211-246.

Brown, C. J., Chen, A. C. N., and Dworkin, S. F. (1989). "Music in the control of human pain," Music Ther. 8, 47-60.

Cabrera, D. (1999). "PsySound: A computer program for psychoacoustical analysis," Proc. Aust. Acoust. Soc. Conf., 47-54.

Cassidy, G. G., and MacDonald, R. A. R. (2009). "The effects of music choice on task performance: A study of the impact of self-selected and experimenter-selected music on driving game performance and experience," Musicae Sci. 13(2), 357-386.

Cassidy, G. G., and MacDonald, R. A. R. (2010). "The effects of music on time perception and performance of a driving game," Scand. J. Psychol. 51, 455-464.

Cepeda, M. S., Carr, D. B., Lau, J., and Alvarez, H. (2006). "Music for pain relief," Cochrane Database of Syst. Rev. 2006(2), CD004843, 62.

Chapin, H., Large, E., Jantzen, K., Kelso, J. A. S., and Steinberg, F. (2008). "Dynamics of emotional communication in performed music," J. Acoust. Soc. Am. 124, 2432.

Chi, C.-Y., Wu, Y.-S., Chu, W.-R., Wu, D. C., Hsu, J. Y.-J., and Tsai, R.T.-H. (2009). "The power of words: Enhancing music mood estimation with textual input of lyrics," 3rd International Conference on Affective Computing and Intelligent Interaction and Workshops, ACII, Amsterdam, The Netherlands, 1-6.

Davis, W. B., and Thaut, M. H. (1989). "The influence of preferred relaxing music on measures of state anxiety, relaxation and physiological responses," J. Music Therapy 26, 168-87.

Dibben, N., and Williamson, V. J. (2007). "An exploratory survey of invehicle music listening," Psychol. Music 35(4), 571-89.

Drake, C., Jones, M. R., and Baruch, C. (2000). "The development of rhythmic attending in auditory sequences: Attunement, referent period, focal attending," Cognition 77, 251-288. 
Eerola, T., Lartillot, O., and Toiviainen, P. (2009). "Prediction of multidimensional emotional ratings in music from audio using multivariate regression models," in Proceedings of 10th International Conference on Music Information Retrieval (ISMIR 2009) (International Society for Music Information Retrieval), 621-626.

Eerola, T., and Vuoskoski, J. K. (2011). "A comparison of the discrete and dimensional models of emotion in music," Psychol. Music 39(1), 18-49.

Ekman, P. and Friesen, W.V. (1998). "Constants across culture in the face and emotion," in Human Emotions: A Reader, edited by J. M. Jenkins, K. Oatley, and N. L. Stein (Blackwell, Malden, MA), pp. 63-72.

Fauerbach, J. A., Lawrence, J. W., Haythornthwaite, J. A., and Richter, L. (2002). "Coping with the stress of a painful medical procedure," Behav. Res. Therapy 40, 1003-1015.

Gabrielsson, A. (2001). "Emotions in strong experiences with music," in Music and Emotion: Theory and Research, edited by P. Juslin and J. Sloboda (Oxford University Press, Oxford, UK), pp. 431-449.

Gabrielsson, A. (2002). "Emotion perceived and emotion felt: Same or different?," Musicae Sci. 123-147.

Gabrielsson, A., and Lindström, E. (2001). "The influence of musical structure on emotional expression," in Music and Emotion: Theory and Research, edited by P. Juslin and J. Sloboda (Oxford University Press, Oxford, UK), pp. 223-248.

Gomez, E., and Herrera, P. (2004). "Estimating the tonality of polyphonic audio files: cognitive versus machine learning modelling strategies," in Proceedings of Fifth International Conference on Music Information Retrieval, Barcelona, Spain (International Society for Music Information Retrieval), pp. 92-95.

Harte, C. A., and Sandler, M. B. (2006). "Detecting harmonic change in musical audio," in Proceedings of Audio and Music Computing for Multimedia Workshop, Santa Barbara, CA (ACM, New York), pp. 21-25.

Helmet, H. M., and Hertel, J.B. (1993). "Pain attenuating effects of preferred versus non-preferred music interventions," Psychol. Music 21, 163-73.

Hevner, K. (1935). "Expression in music: a discussion of experimental studies and theories," Psychol. Rev. 42, 186-204.

Huang, S.-T., Good, M., and Zausniewski, J. A. (2010). "The effectiveness of music in relieving pain in cancer patients: A randomized controlled trial,” Int. J. Nurs. Stud. 47, 1354-1362.

Huron, D. (2006). Sweet Anticipation: Music and the Psychology of Expectation (MIT Press, Cambridge, MA), 476 pp.

Jacobson, A.F. (1999). "Intradermal normal saline solution, self-selected music and insertion difficulty effects on intravenous insertion pain," Heart Lung 28(2), 114-22.

Jiang, D.-N., Lu, L., Zhang, H.-J., Tao, J.-H., and Cai, L.-H.. (2002). "Music type classification by spectral contrast feature," Proc. 2002 IEEE Int. Conf. Multimedia Expo, ICME’02. 1, 113-116.

Juslin, P. N. (2000). "Cue utilization in communication of emotion in music performance: Relating performance to perception,” J. Exp. Psychol. 26(6), 1797-1813.

Juslin, P. (2001). "Communicating emotion in music performance: A review and a theoretical framework," in Music and Emotion: Theory and Research, edited by P. Juslin and J. Sloboda (Oxford University Press, Oxford, UK), pp. 309-340.

Juslin, P. N., and Laukka, P. (2004). "Expression, perception, and induction of musical emotions: A review and a questionnaire study of everyday listening," J. New Music Res. 33, 217-238.

Juslin, P. N., and Västfjäll, D. (2008). "Emotional responses to music: The need to consider underlying mechanisms," Behav. Brain Sci. 31, 559-575.

Knox, D., Cassidy, G., Beveridge, S., and MacDonald, R. A. R. (2008). "Music emotion classification by audio signal analysis: Analysis of selfselected music during game play," in Proceedings of the 10th International Conference on Music Perception and Cognition (ICMPC10), 25-29 August 2008, Sapporo, Japan (Hokkaido University, Hokkaido), pp. 581-587.

Konecni, V.J. (2008). "Does music induce emotion? A theoretical and methodological analysis," Psychol. Aesthetics Creativity Arts 2(2), 115-129.

Krumhansl, C. (2002). "Music: A link between cognition and emotion," Curr. Dir. Psychol. Sci., 11(2), pp. 45-50.

Lamont, A. M., and Webb, R. (2010). "Short- and long-term musical preferences: What makes a favourite piece of music?," Psychol. Music 38(2), 222-241.

Lartillot, O., Eerola, T., Toiviainen, P., and Fornari, J. (2008a). "Multi-feature modeling of pulse clarity: Design, validation, and optimization," International Conference on Music Information Retrieval, Philadelphia (International Society for Music Information Retrieval), pp. 521-526.

Lartillot, O., Toiviainen, P., and Eerola, T. (2008b). "A Matlab toolbox for music information retrieval," in Data Analysis, Machine Learning and
Applications, Studies in Classification, Data Analysis, and Knowledge Organization, edited by C. Preisach, H. Burkhardt, L. Schmidt-Thieme, and R. Decker (Springer-Verlag, Berlin), pp. 261-268.

LeBlanc, A., Colman, J., McCrary, J., Sherrill, C., and Malin, S. (1988). "Tempo preferences of different age music listeners," J. Res. Music Educ. 36, 156-168.

Levitin, D. J. (2006). This is Your Brain on Music: The Science of a Human Obsession [Dutton Adult (Penguin), New York], 320 pp.

Li, T., and Ogihara, M. (2003). "Detecting emotion in music," Proceedings of the Fifth International Symposium on Music Information Retrieval, Baltimore, MD (International Society for Music Information Retrieval), pp. 239-240.

Liu, D., Lu, L., and Zhang, H. J. (2003). "Automatic music mood detection from acoustic music data," in Proceedings International Symposium Music Information Retrieval (ISMIR03) (International Society for Music Information Retrieval), pp. 13-17.

Lu, L., Liu, D., and Zhang, H. J. (2006). "Automatic mood detection and tracking of music audio signals," IEEE Trans. Audio Speech Lang. Proc. 14(1), 5-18.

MacDonald, R. A. R., and Miell, D. (2000). "Creativity and music education: The impact of social variables," Int. J. Music Educ. 36, 58-68.

MacDonald, R. A. R., Mitchell, L. A., Dillon, T., Serpell, M. G., Davies, J. B., and Ashley, E.A. (2003). "An empirical investigation of the anxiolytic and pain reducing effects of music," Psychol. Music 31(2), 187-203.

Madson, A. T., and Silverman, M.J. (2010). "The effect of music therapy on relaxation, anxiety, pain perception and nausea in adult solid organ transplant patients," J. Music Therapy 47, 220-232.

Melzack, R. (1975). "The Mcgill pain questionnaire: Major properties and scoring methods," Pain 1, 277-299.

Melzack, R. (1993). "Pain: Past, present and future," Can. J. Exp. Psychol. 47(4), 615-629.

Melzack, R. (1999). "From the gate to the neuromatrix," Pain (Suppl. 6), 82 (Suppl. 1), S121-S126.

Melzack, R., and Wall, P.D. (1965). "Pain mechanisms: A new theory," Science 150, 971-979.

Meyer, L. B. (1956). Emotion and Meaning in Music (University of Chicago Press, Chicago), 315.

Miell, D., MacDonald R. A. R., and Hargreaves, D. J. (eds.) (2005). Musical Communication. (Oxford University Press, Oxford, UK), 352.

Mitchell, L. A. (2009). "Can music overcome hypervigilance and fear of pain?," 7th Triennial Conference of the European Society for the Cognitive Sciences of Music, 11-16 August, Jyväskylä, Finland, Abstracts and programme, pp. 81.

Mitchell, L. A., and MacDonald, R. A. R. (2006). "An experimental investigation of the effects of preferred and relaxing music listening on pain perception," J. Music Therapy, 43(4), 295-316.

Mitchell, L. A., MacDonald, R. A. R., and Knussen, C. (2008a). "An investigation of the effects of music and art on pain perception," Psychol. Aesthetics Creativity Arts 2(3), 162-170.

Mitchell, L. A., MacDonald, R. A. R., and Brodie, E. E. (2004). "Temperature and the cold pressor test," J. Pain 5(4), 231-238.

Mitchell, L. A., MacDonald, R. A. R., and Brodie, E. E. (2006). “A comparison of the effects of preferred music, arithmetic and humour on cold pressor pain," Eur. J. Pain 10, 343-351.

Mitchell, L. A., McDowall, J. W., and MacDonald, R. A. R. (2008b). "The influence of cognitive style on pain relief using preferred music," Proceedings of the 10th International Conference on Music Perception and Cognition (ICMPC10), 25-29 August 2008, Sapporo, Japan (Hokkaido University, Hokkaido), Abstracts, 9.

Narmour, E. (1990). The Analysis and Cognition of Basic Melodic Structures: The Implication-Realization Model (University of Chicago Press, Chicago), 443 pp.

North, A. C., and Hargreaves, D. J. (1999). "Music and driving game performance," Scand. J. Psychol. 40, 285-292.

North, A. C., and Hargreaves, D. J. (2008). The Social and Applied Psychology of Music (Oxford University Press, Oxford, UK), 394 pp.

North, A. C., Hargreaves, D. J. and Hargreaves, J. J. (2004). "The uses of music in everyday life," Music Percept. 22, 63-99.

North, A. C., Hargreaves, D. J., and McKendrick, J. (1999). "Music and on-hold waiting time," Brit. J. Psychol. 90, 161-164.

Pampalk, E., Rauber, A., and Merkl, D. (2002). "Content-based organization and visualization of music archives," ACM Multimedia 2002, 570-579.

Panksepp, J. (1995). "The emotional sources of "chills' induced by music," Music Percept. 13, 171-207. 
Perlini, A. H., and Viita, K. A. (1996). "Audioanalgesia in the control of experimental pain,” Can. J. Behav. Sci. 28(4), 292-301.

Picard, R. W. (1997). Affective Computing (MIT Press, Cambridge, MA), $275 \mathrm{pp}$.

Pothoulaki, M., MacDonald, R. A. R., Flowers, P. Stamataki, E., Filiopoulos, V., Stamatiadis, D., and Stathakis, C. P. (2008). "An investigation of the effects of music on anxiety and pain perception in patients undergoing haemodialysis treatment," J. Health Psychol. 13, 912-920.

Ritossa, D. A., and Rickard, N. S. (2004). "The relative utility of "pleasantness' and 'liking' dimensions in predicting the emotions expressed by music," Psychol. Music 32(1), 5-22.

Robinson, M. D. (1998). "Running from William James' Bear: A review of pre-attentive mechanisms and their contributions to emotional experience," Cognit. Emotion 12, 697-713.

Roy M., Peretz, I., and Rainville, P. (2008). "Emotional valence contributes to music-induced analgesia," Pain 134, 140-147.

Russell, J. A. (1980). “A circumplex model of affect,” J. Pers. Soc. Psychol. 39, 1161-1178.

Scherer, K. R., and Zentner, M. R. (2001). "Emotional effects of music: Production rules," in Music and Emotion: Theory and Research, edited by P. Juslin and J. Sloboda (Oxford University Press, Oxford, UK), pp. 361-392.

Schoonderwaldt, E., Friberg, A., Bresin, R., and Juslin, P. (2002). "A system for improving the communication of emotion in music performance by feedback learning,” J. Acoust. Soc. Am. 111(5), 2471.

Sethares, W. A. (1998). Tuning, Timbre, Spectrum, Scale (Springer-Verlag, London), $345 \mathrm{pp}$.
Shiffrin, R. M. (1988). "Attention," in Stevens' Handbook of Experimental Psychology, edited by R. C. Atkinson, R. J. Herrnstein, G. Lindzey, and R. D. Luce (Wiley, New York), pp. 739-811.

Sloboda, J. A. (1991). "Music structure and emotional response: Some empirical findings," Psychol. Music 19(2), 110-120.

Sloboda, J. A., and O'Neill, S. A. (2001). "Emotions in everyday listening to music," in Music and Emotion: Theory and Research, edited by P. Juslin and J. Sloboda (Oxford University Press, Oxford, UK), pp. 415-430.

Smith, D. S. (1989). "Preferences for differentiated frequency loudness levels in older adult music listening," J. Music Therapy 26(1), 18-29.

Spielberger, C. D. (1983). State-Trait Anxiety Inventory (Consulting Psychologists Press, Palo Alto, CA).

Terhardt, E. (1974). "On the perception of periodic sound fluctuations (roughness)," Acustica 30(4), 201-213.

Thayer, R. E. (1989). The Biopsychology of Mood and Arousal (Oxford University Press, Oxford, UK), $256 \mathrm{pp}$.

Tzanetakis, G., and Cook, P. (2002). "Musical genre classification of audio signals," IEEE Trans. Speech Audio Process. 10(5), 293-302.

Updike, P. (1990). "Music therapy results for ICU patients," Dimens Crit. Care Nurs. 9(1), 39-45.

Watanabe, S. (1965). "Karhunen-Loeve expansion and factor analysis, theoretical remarks and applications," in Transactions of 4th Prague Conference on Information Theory, Statistical Decision Functions, Random Processes (Academic, New York), pp. 645-660.

Yang, Y. H., Lin, Y. C., Su, Y. F., Chen, H. H., Yang, Y. H., Lin, Y. C., Su, Y. F., and Chen, H. H. (2008). "A regression approach to music emotion recognition,” IEEE Trans. Audio Speech Lang. Proc. 16(2), 448-457. 